\title{
EL PAPEL DEL SUJETO EN LA CONSTRUCCIÓN DEL DESARROLLO
}

Liliana Patricia Torres Victoria 


\title{
RESUMEN
}

\begin{abstract}
El artículo propone la reflexión acerca del desarrollo colocando como eje central del análisis al sujeto como principio orientador del mismo, reconociendo en el sujeto una potencialidad, en tanto que el desarrollo tiene que ver con su misma constitución como sujeto social que piensa y asume posiciones respecto a su contexto en la búsqueda de transformarlo.

En este documento se presentan algunas concepciones teóricas en torno al sujeto social, al sujeto político y a la subjetividad, para finalmente puntualizar en el papel del sujeto en la construcción del desarrollo.
\end{abstract}

niciar una reflexión en torno al papel del sujeto en la construcción del desarrollo implica, necesariamente, ubicar al sujeto en el centro de la discusión como principio orientador del mismo e ir más allá de la visión predominante en el siglo xx acerca del desarrollo, en la cual se relacionan el conocimiento científico, el avance tecnológico y la racionalidad de los mecanismos económicos. Se considera que una sociedad es racional una vez entra en un permanente proceso de innovación tecnológica y cuando busca reducir costos en los ciclos de producción y maximizar su tasa de acumulación capitalista. Al mismo tiempo se asume que estos procesos de desarrollo pueden ser medidos cuantitativamente gracias al uso de indicadores tales como el crecimiento del PIB, la tasa de ahorro,etc. Es decir, desarrollo ha sido asimilado a los conceptos de crecimiento económico, progreso y modernización.

No pretendo señalar que estas condiciones materiales (u objetivas como lo señalan algunos autores) sean menos importantes, el propósito de esta reflexión es reconocer en el sujeto una potencialidad, en tanto que el desarrollo tiene que ver con su misma constitución como sujeto social que piensa y asume posiciones respecto a su contexto en la búsqueda de transformarlo.

El sujeto está inscrito en una multiplicidad de relaciones sociales articuladas no solamente con la producción si no también con el sexo, la raza, la nacionalidad y la territorialidad y de igual forma se expresa en distintas formas de subjetividad. Trasciende la categoría de clase social determinada por la estructura económica y por una 
supraestructura ideológica que define su conciencia social. En tal sentido el sujeto no es predecible, ni es una categoría homogénea desde la cual se puedan identificar fácilmente intereses, pensamientos y acciones. Es importante tener en cuenta su particularidad, ${ }^{1}$ pues desde allí construye múltiples identidades y formas de articularse a lo colectivo.

En este orden de ideas, propongo inicialmente presentar algunas concepciones teóricas en torno al sujeto social, al sujeto político y a la subjetividad. Posteriormente puntualizar en el papel del sujeto en la construcción del desarrollo.

\section{EL DEBATE TEÓRICO RESPECTO A LA CONSTITUCIÓN DEL SUJETO}

Agnes Héller, propone un debate en torno a qué es el sujeto, dónde lo podemos ubicar (en qué contextos y para qué), así como su papel histórico, visto desde diversas corrientes de pensamiento filosófico y sociológico. La diversidad de concepciones lleva a plantearse que su definición es polisémica y en tal sentido puede ser utilizada de diversas formas:

"El término sujeto aparece en los debates contemporáneos franceses y alemanes con los siguientes significados: Punto de vista, individuo, el sujeto de una biografía, el sujeto bermenéutico, el sujeto de conocimiento, el sujeto politico, el sujeto moral, el sujeto persona, personalidad, el yo monocéntrico, el bombre, la autoconciencia, el sujeto como voluntad, además el sujeto abarca todos los casos de sujetos no individuales, no personales, como el sujeto trascendental Kantiano, el espiritu del mundo en begel y también los sujetos personales pero no bumanos, como Dios y los llamados sujetos universales como la historia, el humanismo, los derechos el arte, etc $y$ en algunos casos tienen relación con

el concepto de subjetivo, subjetividad y subjetivismo". (Héller, 2000 :181).

Es significativo este planteamiento, en la medida que nos hace pensar desde donde iniciar una reflexión sobre el sujeto: lo ubicamos en relación con el individuo?, con el ser persona? o con su papel histórico-político? ; para efecto de este trabajo es pertinente ubicarlo en relación con el individuo y su papel histórico-político, ya que es "la singularidad de la propia voz" la que lo coloca en el papel de sujeto con un pensamiento crítico que cuestiona su contexto y las relaciones de poder que se expresan en este. Pero no es sólo el sujeto Universal que surgió con la modernidad, caracterizado por su racionalidad tanto en lo político, como en lo económico y en lo ético, si no, el sujeto con "subjetividades" que se construye de diversas formas y que defiende la particularidad de su voz en ámbitos micro, cotidianos y que busca crear otras identidades.

\footnotetext{
${ }^{1}$ Agnes Héller, desde su obra «Sociología de la vida cotidiana» ubica el particular en condiciones sociales concretas, en sistemas concretos de expectativas, dentro de instituciones concretas, es decir en el mundo de la vida cotidiana desde el cual se piensa y se objetiva desde múltiples formas constituyéndose a si mismo como sujeto.
} 
Los hombres y mujeres modernos se manifiestan como sujetos. Esta manifestación se da en la medida en que pueden elegir sus centros del yo, (u objetos con los cuales se relacionan), cuestionan lo que socialmente se les asigna que deberían ser y eligen sus implicaciones.

Para Héller el sujeto no existe aislado de su entorno, se relaciona con él, lo influye y a la vez es influido por este, los individuos existen. El sujeto es quien hace consciente su existencia, piensa su genericidad su papel en la historia y quien ordena su vida cotidiana con base en esa relación consciente: "El sujeto es un singular que sintetiza en si la unicidad accidental de la particularidad y la universalidad de la genericidad" (Héller, 1987: 19)

En Touraine, la idea de sujeto tiene que ver con pensamiento crítico, cuestionador, y un posicionamiento del individuo frente a la sociedad y al mundo, identifica el surgimiento del sujeto humano como libertad y como creación con dos figuras vueltas la una hacia la otra y cuyo diálogo constituye la modernidad: La racionalización y la subjetivación. Se contradicen pero a la vez se complementan."(Touraine, 2000: 205).

Es decir, en Touraine también encontramos la idea de que el sujeto no sólo tiene que ver con el aprendizaje del pensamiento racional y con la capacidad de resistir a las presiones de la costumbre y del deseo para someterse únicamente al gobierno de la razón, tiene que ver también con las construcciones de sentido que desde su "ser", realice del mundo, interpretaciones que pasan por lo divino, y que a pesar del énfasis en la racionalización, el mundo moderno está cada vez más penetrado por un sujeto que es libertad, es decir que postula como principio del bien el control que el individuo ejerce sobre sus actos y su situación, y que le permite concebir y sentir su conducta como componente de su historia personal de vida, concebirse él mismo como protagonista.

Para el contexto Latinoamericano, la perspectiva de Hugo Zemmelman ${ }^{2}$ con respecto a los conceptos de individuo, sujeto social y sujeto político, permite acercarnos más a la comprensión de la dimensión histórico-política del sujeto en la construcción del desarrollo, porque da cuenta del análisis de nuestra realidad y propone una ruptura entre el papel tradicional de las ciencias sociales (como analista de hechos) y la realidad social; Es decir proponiéndole a la investigación, un papel más activo en la transformación de la realidad social. Es llegar a trascender la función de simple diagnóstico crítico de situaciones, al papel de generar opciones viables frente a lo que se está analizando y asumir posiciones que permitan su transformación.

En esta ruptura, es trascendental la comprensión acerca de la constitución del sujeto, como sujeto social y sujeto político, en una perspectiva que relaciona dialécticamente pasado, presente y futuro, de tal manera que la historia se ubica como un referente de

\footnotetext{
${ }^{2}$ Hugo Zemmelman, teórico que varios autores ubican en una tendencia de la sociología actual conocida como perspectivas de borde o razonamientos de umbral para referirse a formas de conocimiento social que hacen lecturas nuevas sobre la realidad social y que buscan incidir más activamente en su dinámica.
} 
análisis significativo en el proceso de constitución del sujeto. La historia permite construir la identidad de un sujeto en un momento concreto y su papel como actor de esta; la historia es dinámica, genera rupturas y en esta se inscribe el sujeto en su esfuerzo permanente por construirse: "como sujeto buscador de contornos, trasgresor de límites para alcanzar espacios de conciencia y de experiencias más vastas para apropiarse de horizontes nuevos. (Torres, 2000:19). En este sentido se reconoce que el hombre siempre será histórico quiéralo o no, y tenga o no conciencia para asumirlo.

En Zemmelman se identifica el concepto de individuo ligado al pragmatismo y acomodamiento que tienen las personas del pensamiento que se ajusta a formas de reproducción y estabilidad del sistema social, en este sentido, la inteligencia se limita en sus interpretaciones y no se atreve a hacer lecturas que trasciendan la realidad, el individuo es parte de la historia y no generador de historia, es decir el individuo reproduce la realidad social de la cual hace parte sin cuestionarla

Como una alternativa al concepto de individuo, Zemmelman contrapone al Sujeto, cuyo compromiso precisamente es trascender ese anonimato:"La recuperación del sujeto, por consiguiente significa recuperar el sentido de que la historia continua siendo el gran e inevitable designio del hombre, lo que le confiere su identidad como actor concreto porque constituye el contenido de su propia vida" (Zemmelman, 1995, 12)

Esta mirada del sujeto, invita a la construcción de sociedad, es decir a pensarse y asumirse como generador de historia no sólo como parte de la historia. Sólo así se puede establecer el vínculo entre el conocimiento teórico y el sentido de la historia: "Surgen diversos desafíos para enfrentar la tendencia a la ceguera bistórica, la inconsciencia, la inercia mental, la posibilidad de convertirse en víctimas santificatorias de lo que aplasta, deforma y empobrece. De abi que la fecundidad o infecundidad debamos asociarla con la ausencia de proyectos de sociedad (Zemmelman, 1995 :13)

El aspecto central que diferenciaría al sujeto de la categoría de individuo, es básicamente su nivel de conciencia como "generador de historia"; los sujetos sociales reconocen su papel histórico y la potencialidad que tienen para transformar su realidad en historia política. En este sentido la comprensión del sujeto social es distinta para cada contexto histórico, la realidad no existe por fuera del sujeto, no es unívoca, la pluralidad de subjetividades precisamente le da un carácter polivalente: "El análisis de los sujetos supone como requisito tanto no darlos por terminados como concebirlos desde la complejidad de sus procesos constitutivos que tienen lugar en distintos planos de la realidad (Zemmelman, 1995: 13).

El desafío, entonces, consiste en aprehender al sujeto tanto en sus manifestaciones presentes como en sus potencialidades. Los sujetos no son sólo el resultado histórico desde lo económico y tecnológico, son procesos en sí mismos en la medida en que esté presente siempre la potencialidad de su transformación y su capacidad de reactuación sobre las circunstancias que se imponen, es decir desde los lugares que ocupa en la sociedad. 
Es primordial, la conexión entre epistemología y política, pues nos lleva a la pregunta ¿Conocer para que?, ¿conocer para comprender las dinámicas sociales y políticas? $\mathrm{O}$ precisamente para conocer y transformar, dándole una dimensión más compleja al concepto de sujeto social y convertirlo en sujeto político, es decir en un sujeto que no solo se reconoce con conciencia histórica si no que actúa buscando la transformación de su historia. El sujeto político se ubica en la construcción de la identidad colectiva y desde la praxis busca incidir de una manera definitiva en las condiciones estructurales de la realidad social.

La propuesta de Zemmelman asume como criterio fundamental que la función del conocimiento debe consistir en enseñar cómo construir historia. Así el vínculo que se establece entre conocimiento y política permite explicar la unidad de conocimiento e historia.

Sugiere, entonces, que la construcción del conocimiento tiene que considerar las particularidades de la realidad, que son susceptibles de activarse, pero también aquellas dimensiones del sujeto que responden a la necesidad de actuar sobre el contexto.

Recapitulando, hasta aquí, los tres autores identifican en el concepto de sujeto la existencia de un pensamiento critico, cuestionador, que le permite un mayor posicionamiento respecto a su realidad, no obstante la perspectiva de Zemmelman propone diferenciar la categoría de individuo como aquel que reproduce y hace parte de la historia, sujeto social como aquel que adquiere conciencia histórica y construye su sentido como generador de historia, y sujeto político como aquel que además de tener conciencia histórica actúa buscando la transformación de la realidad social. Esta categoría de sujeto político puede compararse en Touraine con la de actor, en el sentido que a ese actor se le obliga a que "actúe" para que se libere: "El sujeto es el deseo del individuo de ser un actor. La subjetivación es el deseo de individuación" (Touraine, 1997: 207) El actor social es igualmente portador del sujeto en sus relaciones interpersonales, las relaciones sociales, las instituciones políticas, las formas de acción colectiva. Pero para descubrirlo no hay que partir ni de las instituciones ni de la comunicación interpersonal, si no de la experiencia vivida del sujeto; el sujeto no es otra cosa que la resistencia, la voluntad y la felicidad del individuo que defiende y afirma su individualidad contra las leyes del mercado y de la comunidad.

\section{SUBJETIVIDAD Y CONSTITUCIÓN DEL SUJETO}

Pero ¿dónde ubicar la discusión sobre la subjetividad y la constitución del sujeto social y político? Zemmelman nos dice que el conocimiento social tendría que incorporarse básicamente más allá de sus especializaciones, en dos ámbitos:

La constitución de la subjetividad social y la articulación de formas discursivas constructoras de relaciones de conocimientos diferentes que busquen una apropiación más inclusiva de la realidad en cuanto esta implique construcción de sentidos. (Zemmelman 1997:23) 
Para Zemmelman la subjetividad social (individual y colectiva), es el plano de la realidad social donde se articulan dimensiones como la memoria, la cultura, la conciencia, la voluntad y la utopía, las cuales expresan la apropiación de la historicidad social a la vez que le confieren sentido y animan su potencialidad. De acuerdo con esto la subjetividad es algo que está presente en todas las dinámicas sociales, en lo micro y lo macro, ya que supone un plano existente y otro por construir (es la subjetividad estructurada y la subjetividad emergente).

Me parece, que el aporte de Zemmelman cuando nos habla de subjetividad, es que nos pone de presente una construcción que va más allá de lo individual. Implica un concepto de lo social a partir de ese dinamismo particular que son los sujetos, los que en últimas se expresan desde los nucleamientos de lo colectivo, ubicándolos en el plano de la relación de ínter subjetividades desde la cual los individuos y los grupos sociales por medio de sus prácticas materiales y simbólicas realizan su propia construcción de la realidad, constituyendo entonces, una subjetividad social particular.

Es importante el papel que le da a lo simbólico, y a la posibilidad de construir y reconstruir representaciones diversas de la sociedad no sólo ubicando al sujeto en una clase social, sino en el contexto de las relaciones ínter subjetivas donde juegan los imaginarios colectivos, las representaciones sociales, las creencias, los mitos, las fantasías.

En este sentido, la idea de nucleamiento de lo colectivo supone entender la dialéctica interna en que descansa la constitución de la subjetividad social intentando encontrar un concepto de subjetividad constituyente; Zemmelman propone una primera dimensión, que se refiere, a retomar las necesidades o mejor la problemática de este movimiento temporo-espacial que lleva a encuadrar este juego de dinamismos en el ámbito delimitado por la memoria y la tradición y de otro por las visiones utópicas, es decir lo que somos, nuestra historia y lo que desearíamos ser (cómo percibimos la necesidad y cómo estamos imaginando su transformación).

La segunda dimensión la denomina opciones de construcción social, en esta se pasa a otro nivel pues se considera la capacidad del sujeto para construir proyectos( y como los lleva a la praxis en la búsqueda de transformación de su entorno): "En este marco el sujeto representa una potencialidad realizada en términos de determinadas alternativas de sentido, esto es, pasa de la pura potencialidad propia de la primera dimensión en la que se contienen múltiples posibilidades de sentido, a la concreción de una alternativa particular de sentido" (Zemmelman, 1997: 13)

Este análisis nos lleva a identificar la inquietud de Zemmelman por ubicar la subjetividad social en el espacio microsocial (en el que se expresan las mediaciones entre individuos y estructuras macro sociales) desde el papel que juegan los sujetos como protagonistas de los acontecimientos presentes y futuros: .."en la dialéctica de lo dado y lo dándose del presente y del futuro, los sujetos son a la vez, producto bistórico y productores de historia, es una doble realidad una aprensible y otra que no lo es" (Ibid). 
Zemmelman hace una propuesta metodológica para estudiar la dinámica de la constitución de la subjetividad y de los sujetos sociales, esta es, reconocer cómo se articula el individuo con los planos en donde se materializan los nucleamientos de lo colectivo, evitando, claro está, reduccionismos a lo psicológico o a lo estructural-social en la búsqueda de explicación de esta articulación. De ahí que se plantee la necesidad de reconceptualizar la idea de sujeto social circunscrita al marco de referencia definido por la categoría de clase, categoría que al reducirse ha dado lugar a una deformación estática y lineal, y por tanto unidimensional, del movimiento de la subjetividad.

Desde esta perspectiva se invita a trascender el análisis de la realidad de los sujetos más allá de los determinismos de la estructura de clases y de las contradicciones generadas por esta, a considerar a los sujetos desde los referentes más inmediatos donde interactúa con la realidad social lo cual nos permite reconocer la potencialidad de que la realidad se construye en oposición a cualquier automatismo histórico, y muestra diferentes horizontes de sentido, en cuanto espacios inciertos por construir.

Siguiendo la línea orientadora de la subjetividad constituyente y el marco de referencia de los nucleamientos de lo colectivo, se podría considerar el siguiente esquema de niveles de lo constitutivo de estos nucleamientos, a saber:

1. Una subjetividad individual. Pensada en función de la inclusión de lo grupal, lo cual permite definir relaciones posibles, las cuales pueden ser de distinto tipo: relaciones entre los individuos que sean de carácter instrumental de lo grupal, relaciones de reciprocidad ,o incluso, de subordinación a la lógica de lo colectivo es decir relaciones con un sentido de responsabilidad social, esto determina cuál es el punto de apoyo de la experiencia de carácter colectivo, sus mecanismos de cohesión y de reproducción.

2. Niveles de nucleamiento de lo colectivo. La apertura de la subjetividad individual hacia lo grupal puede darse en el marco de diferentes ámbitos, tales como: la familia, el grupo de trabajo, la misma comunidad, o bien grupos de referencia que pueden ser externos a esta., esto determinará las posibilidades de provocar la apertura a nuevos espacios de experiencias que sean compartidas. "Uno de los rasgos de estos nucleamientos es que contienen una dirección en potencia; la que impone el poder para privilegiar aquellos que conforman el perfil sico cultural de los individuos (Zemmelman, 1998: 12) Es decir estas realidades más cercanas tienen la potencialidad de definir identidades individuales que reproduzcan formas de actuar determinadas por la estructura social vigente; Pero también tienen la potencialidad de definir identidades colectivas que generen un poder que permita que el sujeto construya realidades de forma más conscientemente definida.

3. Apropiación del contexto. Que poco a poco se va dando en la medida en que el sujeto vaya reflexionando sobre su realidad y se vaya dando lugar la construcción de nuevas experiencias que involucren un papel más consciente en lo colectivo. 
4. Utopía a visión del futuro. En este nivel se interroga si hay o no una voluntad colectiva capaz de sostener una proyección de las acciones hacia el largo tiempo, lo cual implica el reconocimiento de las opciones contenidas en el esfuerzo por transformar la utopía en realidad material.

5. Transformación de la utopía en proyecto viable. Esta sería la culminación del proceso, el momento en el cual el agrupamiento pueda llegar a expresarse en un proyecto, que a diferencia de la pura utopía, no consiste solamente en una necesidad de futuro sino en el imperativo de su construcción.

Cuando Zemmelman nos remite a este esquema metodológico para estudiar la dinámica de constitución de la subjetividad, nos está invitando a develar los elementos constituyentes de la subjetividad y de las dinámicas socio-culturales que expresan el nivel de conciencia de los individuos sobre su realidad desde los ámbitos más cercanos en los cuales se construye como sujeto.

La pregunta que surge es ¿cómo abordar esta subjetividad colectiva en el nivel concreto de la realidad social de la cual hacemos parte?

En este orden de ideas, la aproximación debe hacerse desde la investigación. La subjetividad se expresa en muchas prácticas y discursos en los cuales subyacen elementos ideológicos y políticos que no siempre son tan evidentes. Por eso un proceso investigativo supone problematizar (y problematizarnos) a quienes hacen (o hacemos) parte de una realidad social concreta, además de identificar puntos de activación que permitan trascender el plano meramente comprensivo de esta subjetividad y se configure la praxis, porque sólo allí es donde se hace posible la transformación de la realidad. De esta manera lo potencial se hace posible y se materializa en proyectos orientados a direccionar el presente.

\section{SUJETO Y CONSTRUCCIÓN DEL DESARROLLO}

Con base en el anterior marco de referencia, ¿cuál sería, entonces, la relación entre el sujeto y la construcción del desarrollo? Como punto de partida considero que el desarrollo es un concepto bastante complejo del cual se esbozan diversas posiciones y del cual existe una bibliografía extensa. Sin embargo, para el propósito de este trabajo me interesa indicar un elemento central en estas orientaciones y es que el desarrollo es un proceso que involucra a los sujetos, sus intereses personales, su reflexión sobre las necesidades materiales y la búsqueda permanente de condiciones ,subjetivas y objetivas,que les permitan transformar su entorno.

La transformación del entorno implica necesariamente una posición política, en tal sentido, si la política y el individuo no se piensan recíprocamente cualquier proyecto social perderá su sentido de transformación profunda y radical, es por esto que en el trabajo de los sectores populares se hace necesario replantearse el papel de lo subjetivo y 
la dimensión afectiva implícita en todo proceso de transformación social. Este planteamiento permite justificar la importancia de hablar de sujetos sociales y políticos como categorías que se complementan y desde lo cotidiano se expresan de distintas formas. La práctica social siempre estará atravesada por una intencionalidad que se refleje en el cambio o en la acción para la transformación de realidades concretas.

La comunidad empieza a entenderse no como un hecho debido a una definición externa a ella, si no en función de su dinámica interna, en cuanto a la fuerza de los vínculos, la cohesión del reconocimiento de su propia existencia y de sus posibilidades, el sentido de pertenencia y la unidad de intereses.

En este contexto la subjetividad y el sujeto cobran especial importancia para el análisis de los procesos sociales; las categorías que en un momento histórico permitieron hacer análisis estructurales no son suficientes y es cuando la particularidad debe considerarse para la comprensión de las relaciones sociales, obliga necesariamente a considerar individuo-estructura para tener la visión completa. Es decir la identidad de los agentes sociales no se concibe más como constituida a un nivel único de la sociedad.

Es por esto que el asumir el sujeto como una categoría no homogénea, lleva a que se tenga cuidado de las generalizaciones, las realidades micro tienen que reconstruirse desde la pluralidad de voces de quienes la conforman. La investigación social se redimensiona desde el análisis de lo cualitativo y permite reconocer la ínter-subjetividad en la explicación de los fenómenos.

Como lo señala Martín Hopenhayn, en su texto "La participación y sus motivos":

La motivación central de cada individuo a participar, tiene que ver con ser menos objeto y más sujeto, es decir, cuando la población involucrada en el proceso en cuestión libera potencialidades previamente inhibidas, deja de ser mero instrumento u objeto de otros, se convierte en protagonista de sí en tanto ser social.

En este sentido, el desarrollo tiene que ver con ese proceso particular que vive cada individuo en su proceso de constitución como sujeto, y no solamente con lo que el individuo obtiene por fuera de sí, para garantizar su subsistencia y elevar su calidad de vida. Al pensar sobre sí mismo y tomar conciencia de su papel histórico es "generador de historia". Es decir, al reflexionar sobre sí y su papel en el contexto que lo determina, se da una apropiación del sí mismo y de lo que puede llegar a "ser" y a "hacer" para construir unas mejores condiciones de vida. En esto se traduce lo que Zemmelman propone en la relación necesidad, memoria y utopía: En la dinámica de estos 3 elementos, el sujeto entra en relación con otros y construye subjetividades nuevas, que están influidas por las construcciones de sentido que los otros hacen acerca de su realidad. Es lo que conceptualmente se llamaría una subjetividad social, la cual puede ser nueva en la medida en que las visiones de los otros le aporten. (subjetividad emergente, según Zemmelman). 
La idea del desarrollo como construcción y del sujeto como centro del mismo, busca que se replantee, la visión tradicional del desarrollo "desde arriba" y se consideren los elementos que le aportan desde "abajo", más allá de las técnicas y de los procesos de planificación especializados. De tal manera, que se coloca como un marco de análisis importante el espacio de la vida cotidiana, que es el ámbito más inmediato en el cual se relacionan los sujetos y construyen sus sentidos, significados y representaciones sobre lo social.

El espacio de la vida cotidiana es donde se expresan las mediaciones entre lo micro y lo macro, es decir donde se establecen los puentes con esas instancias que manejan el poder de los recursos, de las decisiones políticas y de la legalidad, y en esa medida contiene toda la potencialidad para re-crearse.

Aquí se reconocen los nucleamientos de lo colectivo (el de lo familiar, de lo grupal y el de lo comunitario) como los referentes desde los cuales se puede llegar a la comprensión del fenómeno y desde donde se pueden impulsar acciones transformadoras.

Los nucleamientos de lo colectivo se constituyen en una propuesta metodológica interesante para la comprensión pero también para la actuación o si se quiere llamar de otra forma, la intervención social, pues es allí donde se construyen y reconstruyen identidades tanto individuales como colectivas, es allí donde se expresan las necesidades y es allí donde los sujetos convierten su pensamiento en acción, que concretamente se refiere a proyectos desde los cuales puedan convertir su utopía en realidad.

Ser más sujeto también nos involucra a quienes, como agentes externos, orientamos procesos tendientes al desarrollo comunitario, implica que para comprender el desarrollo tenemos que romper con la visión tradicional del mismo y comprender a mayor profundidad los procesos histórico-sociales de nuestro contexto tanto local, como regional y nacional.

Replantear nuestro que-hacer como profesionales de las ciencias sociales, en un sentido más dinámico, no sólo como descriptores de realidades, si no como transformadores de las mismas. El siglo xx muestra que la historia es más compleja, de avances, y retrocesos,.La historia vuelve aunque no de la misma manera, lo cual a veces nos impide pensar con claridad para actuar con energía, y preferimos replegarnos. En este sentido, tenemos que rescatarnos como sujetos pensantes, que asumimos posturas claras y somos consecuentes en la acción con este pensamiento.

Un espacio importante, en la relación de subjetividades tanto del profesional como de las personas con las cuales trabajemos, es el educativo. En este debe darse una integración entre lo individual (la historia particular de cada individuo ) y lo epistémico (los conocimientos que va integrando a su vida y que le permiten ser racionales). Es decir, activar un espacio de la subjetividad, que permita potenciar a las personas, que la gente se pueda leer a sí misma desde sus carencias y potencialidades, reconocerse desde la necesidad para poderse liberar, pensarse desde el rol, la función, la institucionalidad 
en el que cada cual se encuentra inscrito y querer ser autónomo para construir una nueva historia.

Finalmente, desde esta perspectiva se constituye en un gran reto para quienes trabajamos en el campo de lo social ubicar al sujeto como eje central de nuestra acción profesional y redimensionar su papel en la construcción del desarrollo, y contribuir a que se haga posible que todo proyecto social sea pensado y logrado por quienes desean la transformación del entorno.

\section{BIBLIOGRAFÍA}

BONETE PERALES ENRIQUe (1995) La faz oculta de la modernidad. Editorial Tecnos, Madrid HÉLLER AGNeS, (1990) "Historia y futuro", !Sobrevivirá la modernidad;, Ediciones Península, Barcelona

TORRES CARRILLO ALFONSO,(1999) Movimientos sociales y organización popular, Universidad Pedagógica Nacional, Bogotá.

TORRES CARRILlo ALFONSO,(1996) Discursos prácticas y actores de la educación popular en Colom-

bia durante la década de los ocehnta. Universidad Pedagógica nacional, Bogotá

TORRES CARRILLO ALFONSO,(2000) "Subjetividad y sujetos sociales en la obra de Hugo Zemmelman"

(2000) Folios, Revista de la Facultad de Humanidades de la Universidad Pedagógica Nacional, Bogotá.

touraine alan, (1997) Podremos vivir juntos. Fondo de Cultura Económica. Bogotá. touraine alan,(2000), Crítica de la modernidad. Fondo de Cultura Económica. Bogotá. Zemmelman hugo,(1996) Problemas antropológicos y utópicos del conocimiento. Jornadas, México. zemmelman hugo, (1995) Determinismos y alternativas en las ciencias sociales de América Latina.

Ediciones nueva sociedad, México

Zemmelman hugo, emma LEÓN, (1997) Subjetividad: umbrales del pensamiento. México.

Zemmelman hugo,(1992) "Los horizontes de la razón" Dialéctica y apropiación del presente

Editorial El hombre, Mexico

ZEMMELMAN HugO, (1992) "Los horizontes de la razón" Historia y necesidad de utopía II. Editorial

El hombre, Mexico

Zemmelman hugo, (1998), Sujeto, existencia y potencia. Antrophos. 